\title{
Race/ethnicity-associated blood DNA methylation differences between Japanese and European American women: an exploratory study
}

Min-Ae Song ${ }^{1 *+} @$, Anna Eames Seffernick ${ }^{2 \dagger}$, Kellie J. Archer ${ }^{2}$, Kellie M. Mori ${ }^{1}$, Song-Yi Park ${ }^{3}$, Linda Chang ${ }^{4}$, Thomas Ernst ${ }^{4}$, Maarit Tiirikainen ${ }^{3}$, Karolina Peplowska ${ }^{5}$, Lynne R. Wilkens ${ }^{3}$, Loïc Le Marchand ${ }^{3}$ and Unhee Lim³

\begin{abstract}
Background: Racial/ethnic disparities in health reflect a combination of genetic and environmental causes, and DNA methylation may be an important mediator. We compared in an exploratory manner the blood DNA methylome of Japanese Americans (JPA) versus European Americans (EUA).

Methods: Genome-wide buffy coat DNA methylation was profiled among healthy Multiethnic Cohort participant women who were Japanese (JPA; $n=30$ ) or European (EUA; $n=28$ ) Americans aged 60-65. Differentially methylated CpGs by race/ethnicity (DM-CpGs) were identified by linear regression (Bonferroni-corrected $P<0.1$ ) and analyzed in relation to corresponding gene expression, a priori selected single nucleotide polymorphisms (SNPs), and blood biomarkers of inflammation and metabolism using Pearson or Spearman correlations (FDR $<0.1$ ).

Results: We identified 174 DM-CpGs with the majority of hypermethylated in JPA compared to EUA ( $n=133)$, often in promoter regions $(n=48)$. Half $(51 \%)$ of the genes corresponding to the DM-CpGs were involved in liver function and liver disease, and the methylation in nine genes was significantly correlated with gene expression for DM-CpGs. A total of 156 DM-CpGs were associated with rs7489665 (SH2B1). Methylation of DM-CpGs was correlated with blood levels of the cytokine MIP1B $(n=146)$. We confirmed some of the DM-CpGs in the TCGA adjacent non-tumor liver tissue of Asians versus EUA.

Conclusion: We found a number of differentially methylated CpGs in blood DNA between JPA and EUA women with a potential link to liver disease, specific SNPs, and systemic inflammation. These findings may support further research on the role of DNA methylation in mediating some of the higher risk of liver disease among JPA.
\end{abstract}

*Correspondence: Song.991@osu.edu

†Min-Ae Song and Anna Eames Seffernick have contributed equally ${ }^{1}$ Division of Environmental Health Science, College of Public Health, The Ohio State University, 404 Cunz Hall, 1841 Neil Ave., Columbus, OH 43210, USA

Full list of author information is available at the end of the article

\section{Introduction}

Racial/ethnic disparities in health are phenotype-specific [1]. While Asian Americans have the longest life expectancy among racial/ethnic populations in the USA based on lower mortality from cardiovascular disease and cancer [2], they are known to have a higher susceptibility to obesity-related metabolic diseases [3]. In the Multiethnic Cohort (MEC) based on five race/ ethnic populations in Hawaii and Southern California original author(s) and the source, provide a link to the Creative Commons licence, and indicate if changes were made. The images or other third party material in this article are included in the article's Creative Commons licence, unless indicated otherwise in a credit line to the material. If material is not included in the article's Creative Commons licence and your intended use is not permitted by statutory regulation or exceeds the permitted use, you will need to obtain permission directly from the copyright holder. To view a copy of this licence, visit http://creativecommons.org/licenses/by/4.0/. The Creative Commons Public Domain Dedication waiver (http://creativeco mmons.org/publicdomain/zero/1.0/) applies to the data made available in this article, unless otherwise stated in a credit line to the data. 
[4], we have observed that Asian Americans tend to develop metabolic syndrome and related diseases, such as type 2 diabetes, starting at a lower level of body mass index (BMI) compared to other racial/ethnic groups [5]. Also, we observed in a magnetic resonance imaging study in a subset of the MEC that Asian Americans have been observed to accumulate disproportionately higher amounts of visceral fat and liver fat across a wide range of BMI or total body fat mass [6]. Consistently, we and others have reported the highest BMI- or total fat mass-adjusted prevalence of non-alcoholic fatty liver disease (NAFLD) and NAFLD-associated hepatocellular carcinoma among Asian Americans [7-9]. Some of these disparities have been attributed to genetic differences, such as the PNPLA3 risk variant for NAFLD (rs738409), which is more common in Asians and Latinos [10]. Still, underlying biological mechanisms on racial/ethnic disparities in health are poorly understood [11].

Epigenetics may be key to elucidate the mechanisms underlying the racial/ethnic differences in health and disease $[12,13]$. DNA methylation is an epigenetic regulator not determined by the DNA sequence. Unlike DNA sequences, the DNA methylome composition is dynamic and influenced by both genetic and environmental factors [14]. For example, altered blood DNA methylation has been observed to be associated with various cardiometabolic diseases and correlated with changes in target tissue DNA methylation, implicating related cellular processes [15]. However, most studies to date for disease associations have been conducted in European descent individuals, and data comparing blood DNA methylation patterns by race/ethnicity are limited [12]. Global blood DNA methylation, as determined by (3H)-methyl acceptance or LINE-1 repeat element methylation, was described to be lower $[16,17]$ or higher $[18]$ in non-European compared to European descent individuals. Recent genome-wide methylation array analyses reported differentially methylated CpGs in African Americans versus European Americans [19].

Considering the potentially important role of DNA methylation in mediating the racial/ethnic health disparities as reviewed above, and based on our previous findings of distinct metabolic disparities among Asian Americans for NAFLD and related liver disease in the MEC $[5-8,11]$, we examined the blood DNA methylome between heathy Japanese American and European American women in the current study. Specifically, we identified differentially methylated CpGs between Japanese Americans and European Americans. Further, we examined how they are associated with corresponding gene expression, related genetic variants, and blood biomarkers of metabolism.

\section{Materials and methods}

\section{Study participants}

Participants for this study were recruited from the Multiethnic Cohort Study (MEC; 1993-current) [4]. As detailed previously [20,21], in 2009-2019, 60 overall healthy, postmenopausal women aged 60-65 were recruited among the MEC participants in Oahu, Hawaii, including self-identified 30 European Americans (EUA) and 30 Japanese Americans (JPA) selected after stratification on age and BMI. This ancillary study within the MEC was a pilot study to explore imaging-based body composition of JPA, who were observed to have higher risks for obesityrelated diseases and cancers in the $\operatorname{MEC}[5,7,8]$ despite their lower mean BMI compared to EUA among generally healthy individuals. The small pilot study included only women due to limited resources and was designed to compare the body composition of generally healthy JPA versus EUA women with comparable BMIs using BMI-stratified recruitment. The participants underwent a detailed body composition assessment, involving a whole-body dual-energy X-ray absorptiometry (DXA) and an abdominal magnetic resonance imaging (MRI) scan, and provided fasting blood and responses to healthrelated questionnaires.

\section{Genome-wide DNA methylation assay and data processing} The Illumina Infinium HumanMethylation450K BeadChips array (Illumina, San Diego, CA) (HM450) was used to profile DNA methylation. Fifty-eight samples (28 EUA and 30 JPA) were available for methylation assays. The QIAamp DNA Mini Kit (Qiagen, Valencia, CA) was used to extract DNA from a fasting blood buffy coat that was stored at $-80{ }^{\circ} \mathrm{C}$. For each sample, $500 \mathrm{ng}$ of DNA was bisulfite converted per the manufacturer's specifications for the HM450 using the EZ DNA Kit (Zymo Research, Irvine, CA). The bisulfite-converted DNA was then hybridized onto the HM450 according to the Illumina Infinium HD Methylation protocol. Images were generated on the Illumina iScan SQ scanner. GenomeStudio (v.2011.1) Methylation module (v.1.9.0) software was used to extract image intensities.

Raw intensity data from the HM450 (.idat files) were read into R version 3.5.2 [22] using the Bioconductor [23] package minfi [24]. Using the probe intensities, $\beta$-values were determined. We used the M-values (logit-transformed $\beta$ ) in our analyses for reduced heteroscedasticity [25]. We started with 485,512 CpGs, and Subset-quantile Within Array Normalization (SWAN) was used to normalize the data [26]. Before further analysis, the data were filtered to remove problematic CpGs. Specifically, Illumina annotation was used to identify $\mathrm{CpGs}$ that overlap with single nucleotide polymorphisms (SNPs) or are within 10 base pairs (bp) of SNPs, which were then 
removed $(\mathrm{n}=89,678)$. We also excluded probes that were in the Y-chromosome, off-target $(\mathrm{n}=31,554)[27,28]$, or had a detection $P$-value $>0.05$. Additionally, CpGs with extremely high methylation $(\beta \geq 0.9)$ or low methylation values $(\beta \leq 0.1)$ were removed $(n=77,715)$ for all samples [29] as these CpGs can be considered to be fully methylated or fully unmethylated, respectively $[29,30]$. After filtering, 285,457 CpGs remained for analysis. The reference genome for this study was GRCh37/hg19 (Human Genome version 19).

Because the methylation assays were performed in two batches (mixed-race/ethnicity per batch), batch effects were adjusted for using the ComBat function [31] (R, sva [32]). We further adjusted for whole blood cell composition to minimize potential confounding $[33,34]$. The proportions of six major cell types in blood DNA $(\mathrm{CD} 8+\mathrm{T}$ cells, CD4 $+\mathrm{T}$ cells, natural killer cells, B cells, monocyte, and granulocyte) were estimated using the estimateCellCounts function (Bioconductor, minfi) [35]. The isometric log-ratio transformation was applied to the matrix of cell compositions, and the transformed values were used as additional covariates in statistical models for associations [36].

\section{Genome-wide transcriptome}

RNA was extracted from the participants' stored whole blood using the PAXgene Kit (Qiagen), and the quality of the RNA was checked on the Agilent Bioanalyzer (Agilent Technologies, Santa Clara, CA), indicating high integrity, with an average RIN of 8.2 (range 7-9). $100 \mathrm{ng}$ of RNA was used for gene expression analysis by the Affymetrix GeneChip Human Transcriptome Array 2.0 (HTA 2.0; Affymetrix Inc., Santa Clara, CA). An Affymetrix GeneChip scanner 3000 with AGCC Software (Affymetrix GeneChip ${ }^{\circledR}$ Command Console ${ }^{\circledR}$ ) was used to scan the arrays. Transcriptome Analysis Console 4.0 (TAC 4.0; Thermo Fisher Scientific, Waltham, MA) was used to assess sample quality, and 2 samples with poor quality were removed, leaving 56 samples for the CpGexpression analysis. The CEL files generated by the arrays were imported into $\mathrm{R}$ using the Bioconductor oligo package [37]. The Robust Multi-Array Average procedure was used to normalize the data and obtain probe set expression summaries [38-40]. The data was annotated using the hta20transcriptcluster.db package [41].

Pairs of expression (transcripts) with cis methylation (probes) were identified for each of the 56 participants (28 JPA, 28 EUA). The HM450 annotation was used to assign gene names to the CpGs, which were then matched to Affymetrix transcripts using Gene Symbol from the hta20transciprtcluster.db annotation. To match gene names listed with different aliases between two data sets, alternative gene names were searched on the
National Center for Biotechnology Information (NCBI) Gene Database (https://www.ncbi.nlm.nih.gov/gene).

\section{Genotyping}

We utilized the Illumina MEGA ${ }^{\mathrm{EX}}$ array. After excluding poor quality SNPs, all SNPs had a call rate $\geq 0.95$ and a replicate concordance 1.00 based on 39 QC replicate samples [11]. Eight well-studied SNPs in metabolic disease-related genes (Additional file 1: Table 1) were selected a priori to be tested for association with identified differentiated CpGs methylation by race/ethnicity.

\section{Blood biomarkers}

In the current analysis, we focused on fasting blood levels of 28 a priori selected biomarkers of lipid metabolism, insulin resistance, liver function and inflammation: specifically, lipid metabolism (high-density lipoprotein (HDL) and total cholesterol, triglycerides (TG)), insulin resistance (homeostatic model assessment for insulin resistance (HOMA-IR)), liver function (alanine and aspartate aminotransferases (ALT, AST), gamma-glutamyl transferase (GGT), cytokeratin 18 (CK18 M30 and M65), and sex hormone-binding globulin (SHBG)), and inflammation (component 3 (C3), high-sensitivity C-reactive protein (CRP), interleukinds and receptors (IL1R $\alpha$, IL-6, IL6R, IL-10, IL-1 $\beta$, IL-2, IL-4, IL-8, IL-5), tumor necrosis factor and receptors (TNF $\alpha$, TNFR1, TNFR2), macrophage inflammatory protein 1 beta (MIP1B), monocyte chemoattractant protein 1 (MCP1), and tissue inhibitor of metalloproteinase 1 (TIMP1)). The priority for these biomarkers were based on their a priori importance for some (e.g., lipid and insulin markers that are used to define the metabolic syndrome) and based on their relevance after finding that a large proportion of differentially methylated CpGs was implicated in liver function and disease (e.g., liver-specific markers, SHBG that we found to be strongly inversely correlated with liver fat and NAFLD [42], and inflammation markers for their relevance in NAFLD and NASH). Analytical methods were reported previously [42]. All assays were performed in one or two batches on the same day, and the included blind duplicate quality control samples showed good reproducibility (coefficient of variation for all assays $2-20 \%[42])$.

\section{Targeted replication of differentially methylated $\mathrm{CpGs}$ between JPA and EUA in the cancer genome atlas (TCGA) liver hepatocellular carcinoma (LIHC) data}

We used the publicly available TCGA-LIHC database (https://portal.gdc.cancer.gov/projects/TCGA-LIHC) to examine the consistency with our differentially 
Table 1 Characteristics of study participant women

\begin{tabular}{|c|c|c|c|}
\hline Characteristics & $\begin{array}{l}\text { All } \\
\text { Mean (SD) or N (\%) }\end{array}$ & $\begin{array}{l}\text { Japanese Americans }(\mathrm{n}=30) \\
\text { Mean }(\mathrm{SD}) \text { or } \mathrm{N}(\%)\end{array}$ & $\begin{array}{l}\text { European Americans }(\mathrm{n}=\mathbf{2 8}) \\
\text { Mean (SD) or } \mathrm{N}(\%)\end{array}$ \\
\hline Age, years & $63.4(1.4)$ & $63.4(1.4)$ & $63.5(1.4)$ \\
\hline Body mass Index, kg/m2 & $26.6(4.6)$ & $26.5(4.7)$ & $26.8(4.5)$ \\
\hline Total body fat mass, kg & $27.0(8.4)$ & $25.5(8.2)$ & $28.6(8.4)$ \\
\hline Liver fat \%, adjusted for total fat* & $4.56(0.51)$ & $5.81(0.98)$ & $3.74(0.55)$ \\
\hline \multicolumn{4}{|l|}{ Smoking history } \\
\hline Never & $37(64 \%)$ & $22(73 \%)$ & $15(54 \%)$ \\
\hline Former & $18(31 \%)$ & $7(23 \%)$ & $11(39 \%)$ \\
\hline Current & $3(5 \%)$ & $1(3 \%)$ & $2(7 \%)$ \\
\hline \multicolumn{4}{|l|}{ Education } \\
\hline 12 years & $5(9 \%)$ & $4(13 \%)$ & $1(4 \%)$ \\
\hline 14 years & $20(34 \%)$ & $13(43 \%)$ & $7(25 \%)$ \\
\hline 16 years & $17(29 \%)$ & $7(23 \%)$ & $10(36 \%)$ \\
\hline 18 years & $16(28 \%)$ & $6(20 \%)$ & $10(36 \%)$ \\
\hline \multicolumn{4}{|l|}{ Blood biomarkers } \\
\hline C-reactive protein (CRP), mg/L & $2.0(3.3)$ & $1.7(3.6)$ & $2.4(2.9)$ \\
\hline $\begin{array}{l}\text { Macrophage inflammatory protein-1 beta } \\
\text { (MIP1B), pg/mL }\end{array}$ & $30.7(12.9)$ & $36.0(13.9)$ & $25.0(8.8)$ \\
\hline Triglycerides (TG), mg/dL & $93.0(75.6)$ & $107.1(95.9)$ & $77.8(41.5)$ \\
\hline Insulin, mIU/L & $8.3(8.1)$ & $10.5(9.4)$ & $6.0(5.9)$ \\
\hline Alanine Aminotransferase (ALT), U/L & $31.8(16.1)$ & $34.4(18.4)$ & $29.0(13.0)$ \\
\hline \multicolumn{4}{|l|}{ Blood cell compositions } \\
\hline CD8 + T cells & $0.05(0.04)$ & $0.05(0.03)$ & $0.05(0.04)$ \\
\hline CD4+T cells & $0.15(0.06)$ & $0.15(0.06)$ & $0.16(0.07)$ \\
\hline Natural killer cells & $0.09(0.05)$ & $0.10(0.05)$ & $0.08(0.05)$ \\
\hline B cells & $0.06(0.03)$ & $0.08(0.03)$ & $0.05(0.02)$ \\
\hline Monocytes & $0.09(0.02)$ & $0.09(0.02)$ & $0.10(0.02)$ \\
\hline Granulocytes & $0.57(0.08)$ & $0.56(0.08)$ & $0.58(0.07)$ \\
\hline
\end{tabular}

* For 46 participants (20 Japanese Americans, 26 European Americans) with MRI measurements

methylated CpGs between JPA and EUA. The Level 1 genome-wide DNA methylation data for the adjacent non-tumor tissues (24 samples from men, 16 samples from women) from 6 Asian LIHC cases (of no specified Asian subtype available) and 34 EUA cases were analyzed. The data were normalized using SWAN, and batch effects were removed by adjusting for batches before the data analysis.

\section{Statistical analyses}

All statistical analyses were performed in $\mathrm{R}$ version 3.5.2, relevant Bioconductor version 3.7 packages, and the Partek Genomics Suite ${ }^{\mathrm{TM}} 6.6$ (St. Louis, MO). To compare the descriptive characteristics of JPA versus EUA, Welch's $t$ tests were used for continuous traits and Fishers' exact test for categorical traits. The total fat-adjusted liver fat values were obtained in general linear models (ANCOVA).

\section{Identification and characterization of differentially methylated C $p$ Gs between JPA and EUA}

CpGs differentially methylated by race/ethnicity (DMCpGs) were identified in linear regression of the $M$-value for each CpG on race/ethnicity (JPA vs. EUA) (R Bioconductor, limma [43]). Significant CpGs (Bonferronicorrect $P<0.1$ ) were visually examined for their ability to separate JPA from EUA by applying hierarchal clustering with Unweighted Pair Group Method with Arithmetic Mean (UPGMA; R, hclust). The CpGs were also examined in a volcano plot, showing the mean methylation difference in each for JPA versus EUA $(\Delta \beta$; positive for hypermethylation and negative for hypomethylation in JPA vs. EUA) plotted against $-\log _{10}[P$-value $]$.

Welch's $t$ test was used to compare the mean methylation of the DM-CpGs between JPA and EUA $(P<0.05$ for significance) stratified by categories defined by the genomic location of the $\mathrm{CpG}$ or proximity to $\mathrm{CpG}$ islands. The genomic location (promoter, non-promoter, 
or intergenic) was defined according to the Illumina's annotation file (https://support.illumina.com/content/ dam/illumina-support/documents/downloads/produ ctfiles/methylationepic/infinium-methylationepic-manif est-column-headings.pdf). Finally, the DM-CpGs were examined in Ingenuity Pathway Analysis (IPA) (Qiagen) to assess their likely functional involvement.

Separately, we compared the mean of $285,457 \mathrm{CpGs}$, as global methylation and the mean DNA methylation age (Hannum's blood epigenetic clock [44]) between JPA and EUA using Welch's t test.

\section{Correlation of differentially methylated CpGs by race/ ethnicity with gene expression and blood biomarkers}

Pearson correlation was used for the association of DM-CpGs with their corresponding gene expression, using Benjamini and Hochberg False Discovery Rate $($ FDR $)<0.1$ for significance. Spearman correlation was used to determine if the DM-CpGs were associated with each blood biomarker (FDR $<0.1$ for significance).

\section{Association between differentially methylated CpGs by race/ ethnicity and SNPS}

Pearson's chi-square test was used to determine whether allele distribution differed by ethnicity for each SNP. To determine if genetic variants in metabolic disease-related SNPs were associated with DNA methylation at the DMCpGs, ANOVA models were used. For each SNP, samples with missing data were removed, and an indicator variable was created that was 1 if the sample contained at least one risk allele. A simple ANOVA model was fit for each DM-CpG, with DNA methylation as the outcome and risk allele indicator as the covariate. $\mathrm{FDR}<0.1$ was used to determine significance.

\section{Comparison of differentially methylated CpGs by race/ ethnicity with TCGA liver tissue methylation}

Once we found that the majority of the DM-genes between JPA and EUA were enriched in liver function/ liver diseases, we utilized TCGA data to: (1) examine whether these CpGs were similarly differentially methylated in non-tumor liver tissue of Asians versus EUA and (2) examine whether these CpGs were differentially methylated between liver tumor and adjacent non-tumor tissue, in order to explore their potential involvement in liver tumorigenesis. To identify the CpGs differentially methylated between Asians $(n=6)$ versus EUA $(\mathrm{n}=34)$ in their non-tumor liver tissue, we used threeway ANOVA with adjustment for age and sex. To identify the CpGs differentially methylated between hepatocellular carcinoma tumors and adjacent non-tumor tissues ( $\mathrm{n}=40$ pairs), we performed two-way ANOVA with adjustment for pairs (FDR $<0.1$ for significance).

\section{Results}

\section{Participant characteristics}

As reported previously [20], JPA and EUA women were all post-menopausal and had similar age and BMI distributions by study design, JPA had a higher level of total adiposity-adjusted liver fat compared to EUA (Table 1). JPA and EUA did not differ in smoking history, education, blood levels of CRP, TG, and ALT in Table 1. However, JPA had higher blood concentrations of MIP1B (36.0 pg/ $\mathrm{mL}$ vs. $26.7 \mathrm{pg} / \mathrm{mL}$ in EUA; $P=0.006)$ and insulin $(10.5$ vs. $6.0 \mathrm{mIU} / \mathrm{L} ; P=0.03$ ) compared to EUA.

\section{Identification of differentially methylated CpGs between JPA and EUA}

When compared for the mean methylation level for all CpGs analyzed, a value that can be taken as a measure of global methylation, we observed a significantly higher mean methylation level in JPA (mean $\beta=0.565$ ) compared to EUA (mean $\beta=0.556)(P=0.00018)$ (Fig. 1A). For the blood epigenetic clock index, JPA had a higher mean DNA methylation age (64.19) than EUA (62.69), but the difference was not statistically significant (data not shown).

From the locus-specific analysis, we identified 730 differentially methylated CpGs (DM-CpGs) between JPA and EUA (Bonferroni $P<0.1$ ), which were reduced to 174 DM-CpGs after adjusting for blood cell type composition: 160 of the 174 were found among the 730 (Additional file 2: Table 2). With respect to the cell proportions, JPA had significantly higher B cells and monocytes than EUA $(P<0.05)$, but did not differ for CD8 + T cells, CD4 + T cells, natural killer cells, and granulocytes (Table 1). None of the DM-CpGs were associated with the level of education.

The majority of the DM-CpGs $(n=133,76 \%)$ were hypermethylated in JPA compared to EUA, as shown in the volcano plot (Fig. 1B) and hierarchical clustering (Fig. 1C). The DM-CpGs were spread across all autosomes, as shown in the Manhattan plot (Fig. 1D). The top ten DM-CpGs based on statistical significance are presented in Fig. 2. Five CpGs were associated with genes HHLA2, LOC91948, WDR16, VWA1, and OCA2, as well as intergenic CpGs (cg12407057, cg11156891, cg07073561, cg00587301, and cg00695177). A full list of the 174 DM-CpGs and their genomic characteristics is provided in Additional file 3: Table 3.

\section{Characterization of differentially methylated CpGs between JPA and EUA}

We compared the mean methylation of DM-CpGs for JPA compared to EUA within each functional location category. We found significantly more hypermethylation 


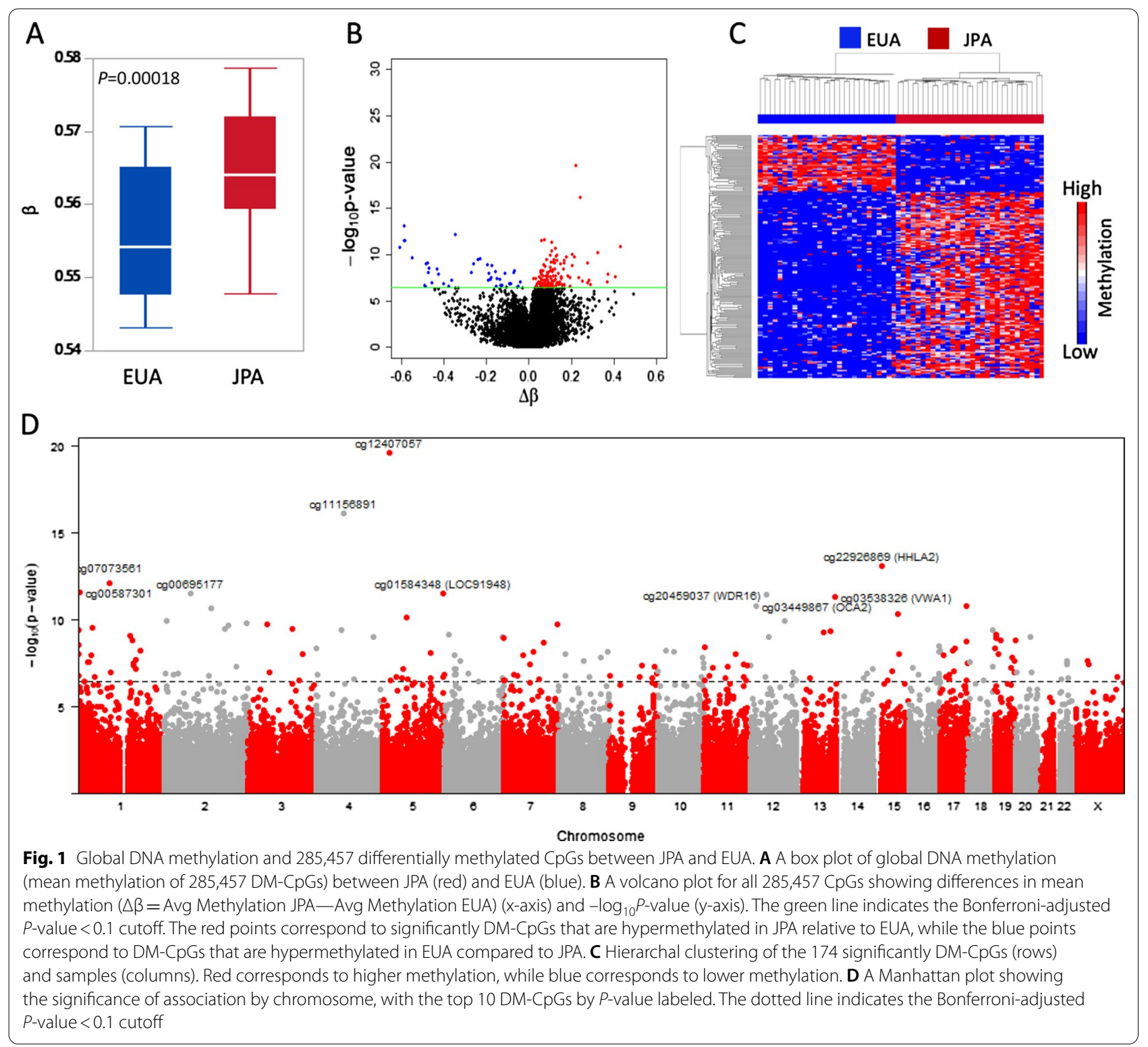

of DM-CpGs in JPA (36\% vs. 15\%) within the promoter region but did not detect differences within the non-promoter or intergenic region (Additional file 4: Table 4). Figure 3 shows a further comparison of mean methylation of the DM-CpGs in JPA versus EUA stratified by $\mathrm{CpG}$ island-related regions (CpG islands, Northor South-shelves, North- or South-shores, and open sea regions) within each genomic location (promoter, non-promoter, and intergenic). Across all CpG islandrelated regions, mean methylation varied by race/ethnicity the most in promoters. There was a large variation in the DM-CpG methylation positioned in N-shelf, among EUA for promoter or non-promoter location and among JPA for intergenic location. Mean methylation was significantly higher among JPA for 12 of the 15 regions, whereas it was higher among EUA for only $\mathrm{S}$-shore in the intergenic region $(P<0.05)$.

\section{Correlation of differentially methylated CpGs between JPA and EUA with corresponding gene expression}

Of the 174 DM-CpGs, 116 (67\%) corresponded to 111 unique genes, resulting in 147 DM-CpG-transcript pairs (Additional file 5: Table 5). Eleven CpG-transcript pairs (9 unique $\mathrm{CpGs}$ and 10 unique transcripts) were significantly correlated at $\mathrm{FDR}<0.1$ (Fig. 4). AFAP1, CSMD3, GATM (3 transcripts), KIAA0748, SH3BP4, and SOX6 were negatively correlated, and CCDC66 (2 transcripts) and MRPL15 were positively correlated. The 

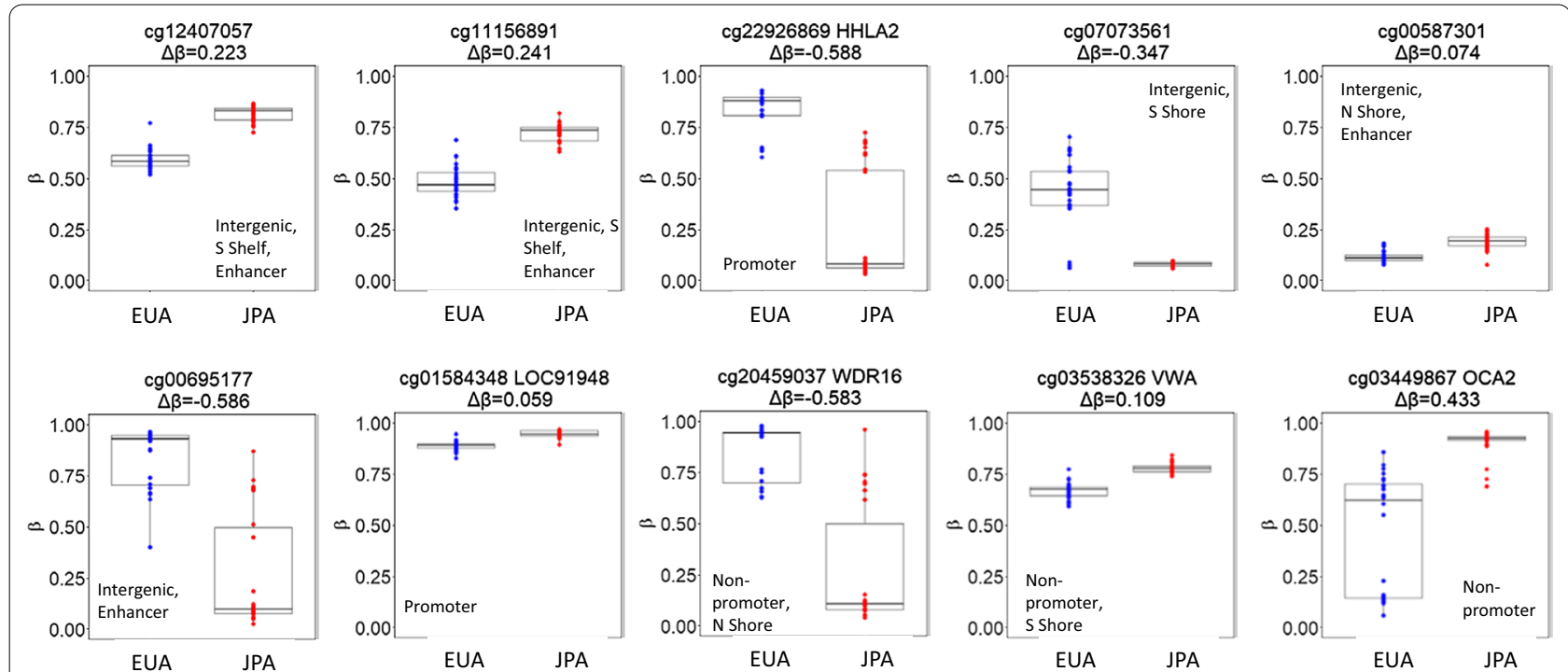

Fig. 2 Dot plots of methylation ( $\beta$-value) between JPA and EUA for the top ten significantly differentially methylated CpGs by $P$-value. All have $P$-value $<1.5 e-11($ FDR $<0.1)$. Blue points indicate EUA $(n=28)$, and red points indicate JPA $(n=30)$. $\triangle \beta$ is defined as average methylation in JPAaverage methylation in EUA. $\triangle \beta>0$ corresponds to hypermethylation in JPA relative to EUA, while $\triangle \beta<0$ corresponds to hypermethylation in EUA relative to JPA

strongest correlation was found for AFAP1 (cg13534536TC04001014.hg.1) ( $\mathrm{r}=-0.83)$, driven by the correlation among EUA $(r=-0.77)$, while no significant correlation was detected in JPA $(\mathrm{r}=-0.031)$. Also, the CpGtranscript correlation for KIAA0748 was strong in JPA $(\mathrm{r}=-0.72)$ but not in EUA $(\mathrm{r}=0.042)$.

\section{Potential biological roles of differentially methylated CpGs between JPA and EUA}

We examined 102 of the 111 unique genes corresponding to the DM-CpGs in the IPA analysis and found that the majority of these genes $(52,51 \%)$ were functionally enriched in liver function/diseases, including liver inflammation, hyperplasia, proliferation, cirrhosis, and regeneration. These are summarized in Additional file 6: Table 6.

\section{Associations between differentially methylated $\mathrm{CpGs}$} between JPA and EUA and metabolic disease-related SNPs We further explored the association between the DMCpGs and eight previously reported metabolic disease-related SNPs (Additional file 1: Table 1). Among 174 DM-CpGs, 156 were significantly associated with rs7498665 (SH2B1), 3 with rs738409 (PNPLA3), and 49 with rs29941(KCTD15) at FDR $<0.1$ (Additional file 7: Table 7). Some associations remained significant even after adjusting for race/ethnicity. These corresponded to 3 CpGs (cg22216157 [PTPRN2], cg24524099 [PTPRN2], and $\operatorname{cg} 02903756$ [CASZ1]) for rs738409 and 2 CpGs (cg07073561 [intergenic] and cg07863524 [OR3A4]) for rs29941.

Correlation of differentially methylated CpGs between JPA and EUA with blood biomarkers of inflammation and metabolism

We further investigated the association of the methylation levels of 174 DM-CpGs with 28 blood biomarkers of inflammation, lipids, insulin resistance, and liver function. There were 183 significant CpG-biomarker associations, involving 149 CpGs (86\% of DM-CpGs) and 5 biomarkers at FDR $<0.1$ : MIP1B $(\mathrm{n}=146)$, HOMA-IR $(\mathrm{n}=18)$, ALT $(\mathrm{n}=1)$, IL-1 $(\mathrm{n}=17)$, and IL-5 $(\mathrm{n}=1)$ (Additional file 8: Table 8). Thirty-one CpGs were significantly correlated with multiple biomarkers, which were mostly associated with genes $(n=27 ; 87 \%)$. The majority of the CpG-biomarker associations (146/183, 80\%) involved MIP1B, and top correlated CpGs were associated with genes, SEPT9, HHAL2, OR3A4, NIPA1, and PTPRN2. None of the DM-CpGs were statistically associated with the other 23 biomarkers.

\section{Consistency of differentially methylated CpGs between JPA and EUA in TCGA-LIHC data}

Given that the DM-CpGs between JPA and EUA are enriched in liver function and liver diseases/cancer, the 174. DM-CpGs were queried in the TCGA-LIHC data (Additional file 9: Table 9) to examine consistency in adjacent non-tumor tissues. We further explored the 

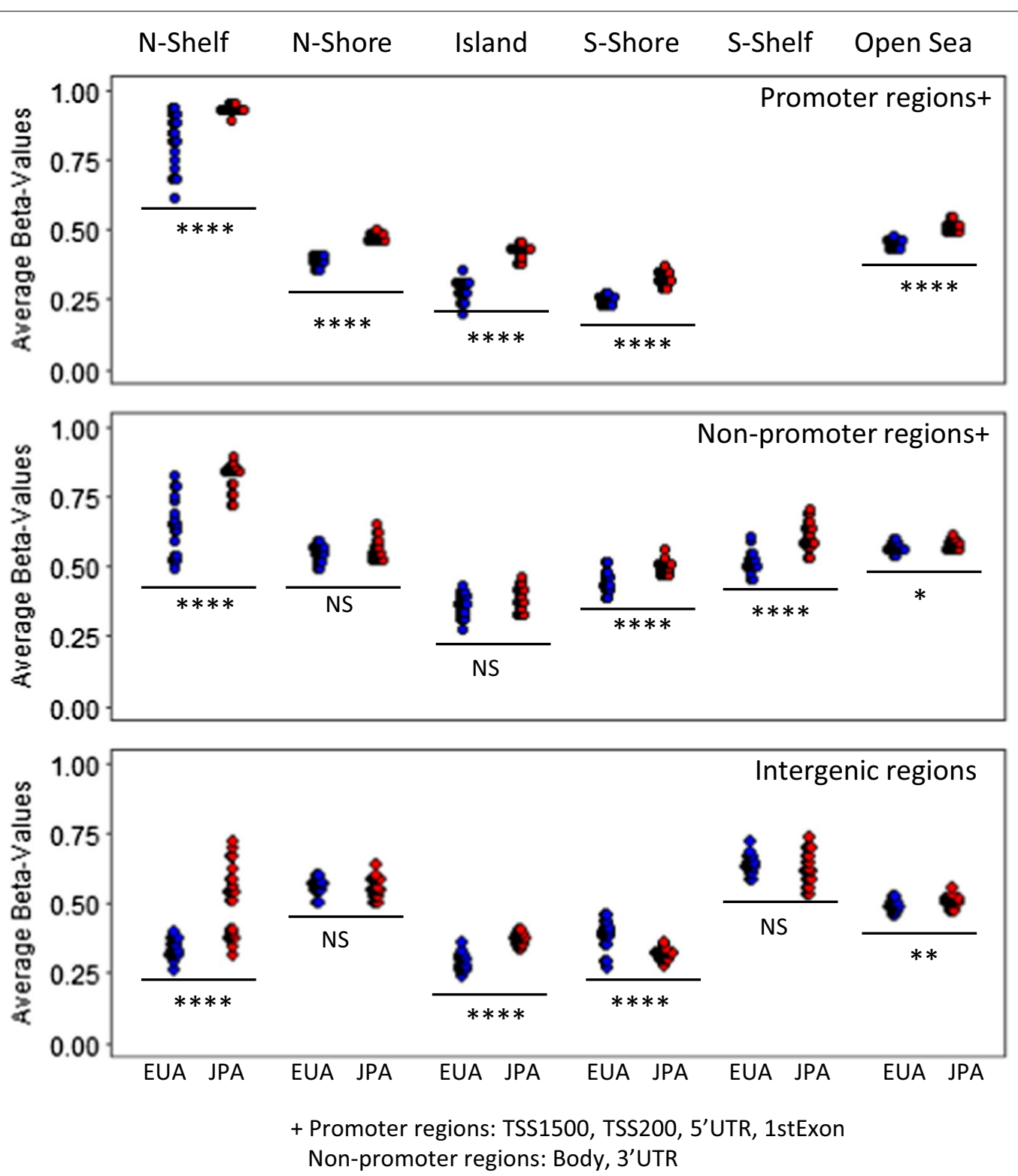

Fig. 3 Methylation levels stratified by location of significant differentially methylated CpGs between JPA and EUA. Average methylation levels for each sample within $\mathrm{CpG}$ islands and surrounding regions are shown in promoters (top panel), non-promoters (middle panel), and intergenic regions (bottom panel). Blue corresponds to EUA, and red corresponds to JPA. Differences in average methylation for EUA and JPA in each region were tested using unpaired Welch's $t$ test $\left({ }^{* * *} P<0.0001,{ }^{* * *} 0.0001\right.$ to $0.001,{ }^{* *} 0.001$ to $0.01,{ }^{*} 0.01$ to 0.05 , NS not significant)

possible involvement of the DM-CpGs in tumorigenesis by comparing the CpGs between liver tumor and adjacent non-tumor tissue in the TCGA data. Thirtyeight CpGs (22\%) were differentially methylated in the adjacent non-tumor tissues of Asian and EUA cases of hepatocellular carcinoma (FDR $<0.1)$, adjusted for age and sex, with 35 (92\%) of them in a consistent racial/ ethnic pattern as in our blood DNA analysis (Additional file 10: Table 10). For several example CpGs shown in Fig. 5A, the left panel depicts the Asian versus
EUA difference. Of the 174 DM-CpGs, 110 CpGs (63\%) were differentially methylated in paired tumor versus adjacent non-tumor tissues (pair-adjusted FDR $<0.1$ ): the right panel of Fig. 5A depicts the tumor- non-tumor tissue difference. A heatmap of the $110 \mathrm{CpGs}$ shows overall lower methylation of these CpGs in tumors compared to adjacent non-tumor tissues (Fig. 5B): the top five hypermethylated genes were GLRX, WNT9B, SEPT9, KIAA00284, and PPYR1, and the top 

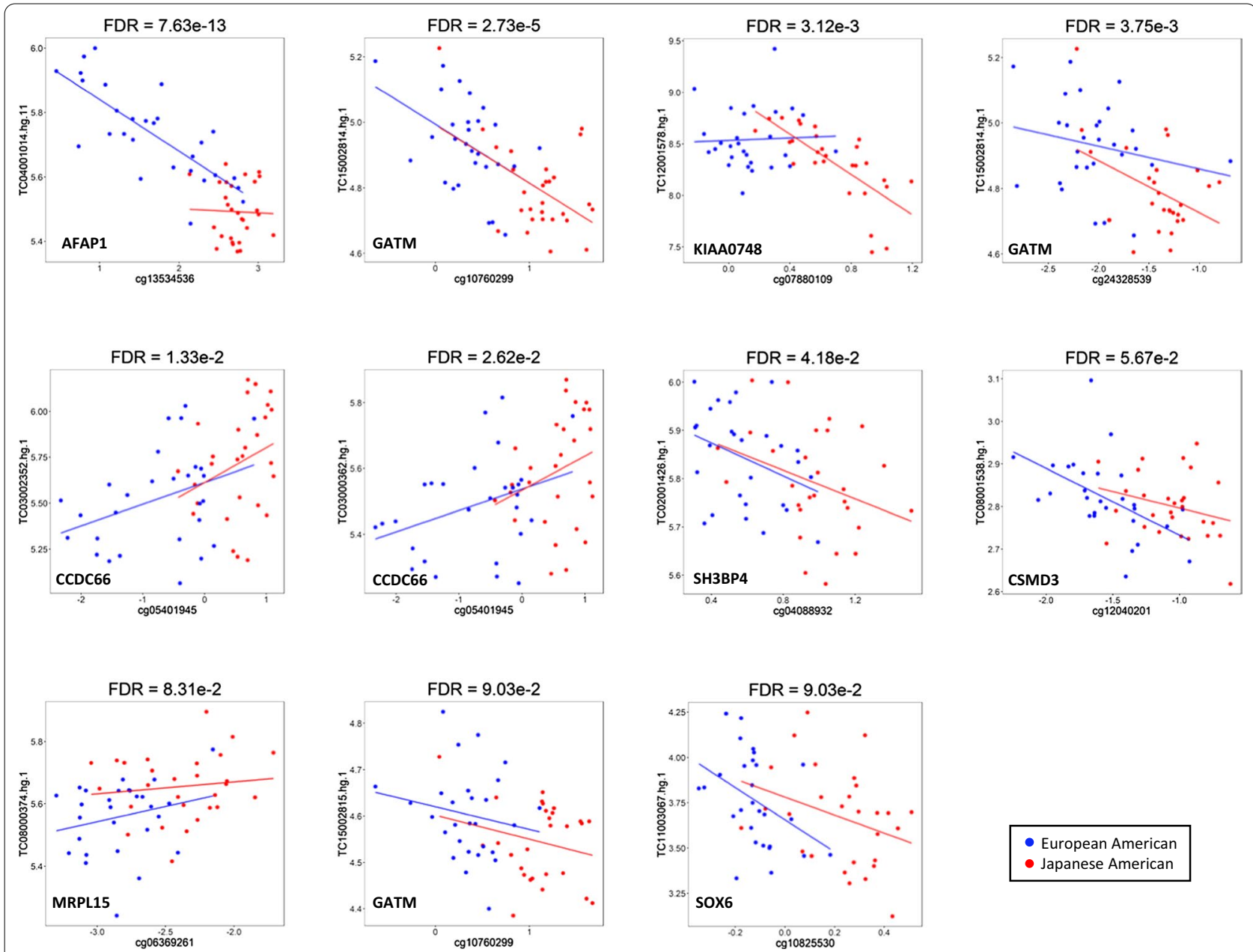

European American

- Japanese American

Fig. 4 Scatter plots of transcript expression ( $\log _{2}$ ) versus DNA methylation (M-value) with significant Pearson correlation by FDR $<0.1$. The correlation FDR is indicated at the top of each plot. The blue points and lines correspond to EUA $(n=28)$, and the red points and lines correspond to $J P A(n=28)$

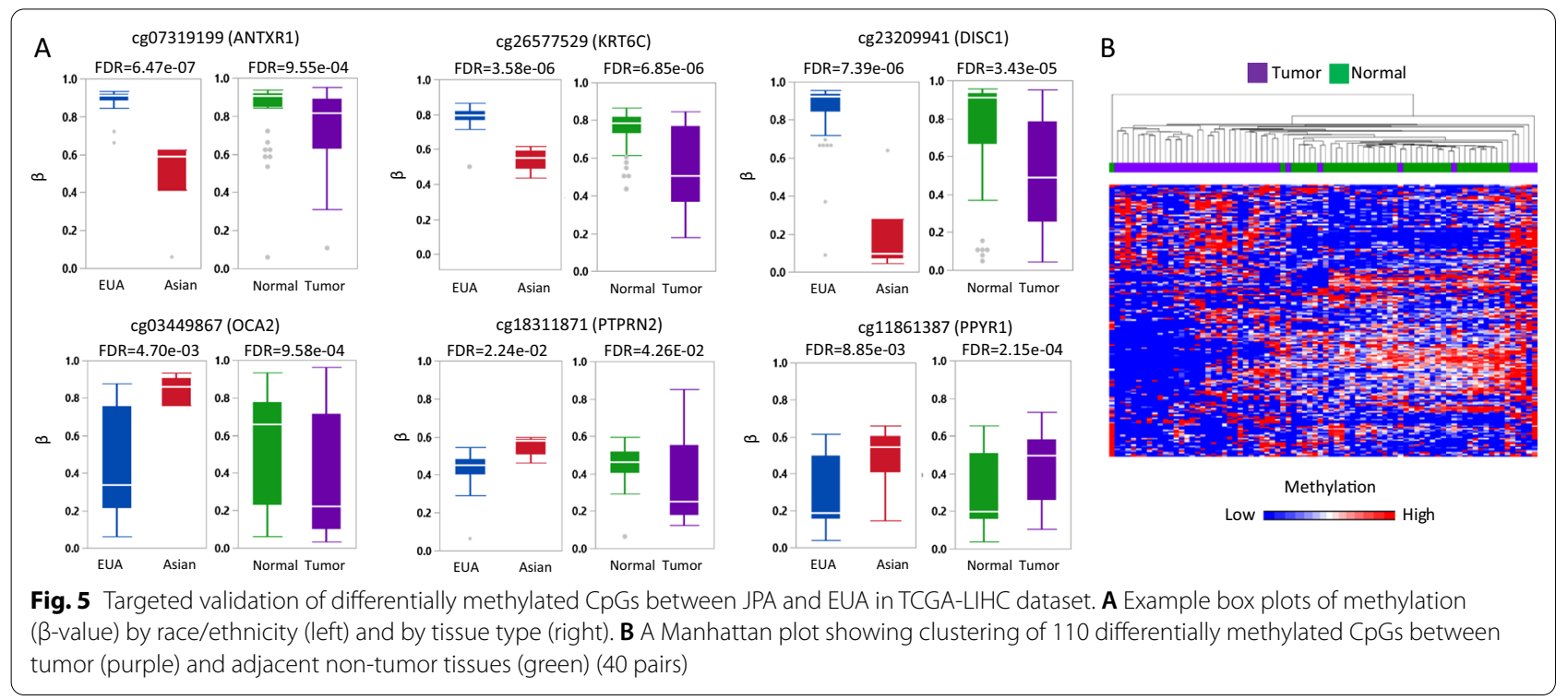


five hypomethylated genes were KIAA0748, PAEP, PCDH15, PTPRN2, and DUSP27 (Additional file 11: Table 11).

\section{Discussion}

While some evidence points to racial/ethnic differences in blood DNA methylation and a postulated role for these differences in racial/ethnic health disparities [12, 45], data are still very limited on racial/ethnic comparisons and their biological implications. In this cross-sectional study, we found blood genome-wide differential methylation between JPA and EUA for 174 CpGs among generally healthy post-menopausal women. The majority of CpGs differentially methylated by race (DM-CpGs) $(76 \%)$ were hypermethylated in JPA compared with EUA and highly enriched in promoter regions. The methylation levels of only a small subset of the DM-CpGs were positively or negatively correlated with their corresponding gene expression. Gene enrichment analysis for DM-CpGs revealed that the majority of the genes for these CpGs were involved in liver function and liver diseases, which may explain the substantially higher susceptibility of JPA to liver disease and liver cancer reported in this [20] and larger studies [6, 7]. Notably, most of the DM-CpGs were also associated with a blood biomarker of inflammation MIP1B. In the TCGA-LIHC (hepatocellular carcinoma) dataset, we confirmed some of the DM-CpGs in the TCGA non-tumor liver tissue of Asians compared to EUA. A large proportion of the DM-CpGs were also differentially methylated between liver tumor and nontumor tissues.

Our study adds to the previous evidence for racial/ ethnic differences in DNA methylation as reflecting underlying biological mechanisms possibly underlying some racial/ethnic health disparities.[17, 45-48]. Most past studies analyzed global DNA methylation, such as LINE-1 methylation, with inconsistent findings in small numbers of African or Hispanic ancestry [12]. In our study using a genome-wide methylation array, the mean methylation levels of all CpGs, a marker of global methylation, were significantly higher in JPA compared to EUA.

The majority of our DM-CpGs were involved in liver function and liver disease. Of the genes with DM-CpGcorrelated gene expression, AFAP1 and KIAA0748 in particular were distinctly expressed in JPA versus EUA. $A F A P 1$, found to be expressed at lower levels in JPA with hypermethylation of cg13534536 in this study, codes for actin filament associated protein 1, and its antisense RNA promotes liver tumor cell proliferation, indicative of a poor diagnosis [49]. KIAA0748 is also known as TESPA1, and its deletion was detected in cirrhotic liver tissue [50]. Also differentially methylated and expressed in this study was SOX6, a transcription factor, acting as an activator of adipogenesis [51]. In the TCGA-LIHC dataset, we further found significantly differential methylation between tumor and adjacent non-tumor tissue for AFAP1, KIAA0748, and SOX6. Additional studies are needed to understand whether the liver diseases related to DM-genes contribute to the risk and progression of liver diseases.

Genetics may directly or indirectly explain an important part of the epigenetic differences by race/ethnicity. Here, we found $90 \%$ of the DM-CpGs between JPA and EUA to be significantly associated with rs7498665 (SH2B1). Also, we identified $3 \mathrm{CpGs}$ to be associated with rs738409 (PNPLA3) and 49 for rs29941(KCTD15). SH2B1 is a well-known metabolic regulator related to obesity and liver lipid metabolism [52, 53], and rs7498665 is associated with visceral fat [54]. PNPLA3 genetic variant, rs738409, is a significant genetic risk factor for hepatic steatosis by accumulating high lipid droplets $[55,56]$. Although the role of KCTD15 is unclear in liver diseases, this gene is an obesity-related gene [57], and its genetic variation (rs29941) was significantly associated with weight changes [58] and fasting plasma glucose level [59]. As it is difficult in this small study for genetic polymorphisms to tease out independent SNP-CpG correlations from racial/ethnic differences in minor allele frequency, further study is warranted to investigate the role of DNA methylation in mediating racially/ethnically differential genetic susceptibility for metabolic diseases.

It is important to note that, in addition to the initial exclusion of SNPs associated probes in order to avoid potential risks of SNPs in the probe regions, we evaluated whether our 174 DM-CpGs overlap with any SNPs from 1000 Genomes available in the dbSNP database. We searched the chromosomal location of each CpG site and found that 21/174 (12.1\%) overlap with the SNPs of 1000 Genomes. Of these 21, only 9 SNPs $(9 / 174,5.2 \%)$ had slightly different allele frequencies between East Asians and Europeans (e.g., $\mathrm{C}=0.9990 / \mathrm{T}=0.0010$ vs $\mathrm{C}=1.0000 / \mathrm{T}=0.0000$ for most cases). However, methylation levels at these $9 \mathrm{DM}-\mathrm{CpGs}$ had a continuous distribution, indicating no SNP effects on methylation at these CpGs.

Finally, we found eight DM-CpGs that were consistently associated with corresponding gene expression, important genetic variation (rs7498665, SH2B1), and systemic inflammation (MIP1B): these were cg12040201 in CSMD3, cg04088932 in SH3BP4, cg24328539 in GATM, cg05401945 in CCDC66, cg07880109 in KIAA0748, cg10760299 in GATM, cg10825530 in SOX6, and cg13534536 in AFAP1. CSMD3 is considered a driver gene in liver cancer [60]. Although the biological function of CSMD3 has not been fully understood, this gene is associated with alcohol exposure [61] and its 
genetic alteration is associated with morbid obesity [62]. SH3BP4 is a potential tumor suppressor, and its methylation is related to impaired insulin signaling [63]. Elevated blood levels of proinflammatory cytokine MIP1B, also known as CCL4, are observed in liver inflammation and fibrosis $[64,65]$. Future studies on a larger scale may be able to disentangle the genetic-epigenetic-phenotype associations by race/ethnicity.

This study has several strengths. It was nested within a long-term cohort providing the advantage of having wellcharacterized participants with various types of data. Our study integrated analysis of gene enrichment, gene expression, SNPs, blood biomarkers, and the TCGA. The consistency in findings across these analyses are supportive of the potential role of race/ethnicity-associated DNA methylation patterns in metabolism. However, this initial exploratory study had clear limitations, including a small sample size and the cross-sectional study design. While we had sufficient power to identify a number of significant DM-CpGs and detect their association with some biomarkers, larger multiethnic studies including both sexes are warranted to investigate racial/ethnic DNA methylation profiles more systematically. To identify potential drivers of DM-CpGs by race/ethnicity, additional studies for the effects of the environment, lifestyle, nutrition, and individual and contextual socioeconomic status on these associations should be performed with relevant health outcomes more carefully.

In conclusion, our findings provide supportive evidence that differential blood DNA methylation across racial/ ethnic populations may represent epigenetic mechanisms underlying phenotype differences and disparities. Larger and more diverse studies are warranted to explore these relationships further.

\footnotetext{
Abbreviations

FDR: Benjamini and Hochberg False Discovery Rate; EUA: European Americans: CpG: CpG site (cytosine-guanine); DM: Differentially methylated; JPA: Japanese Americans; MEC: The Multiethnic Cohort Study; SNP: Single nucleotide polymorphism; TCGA: The Cancer Genome Atlas; TSS: Transcription Start Site; UTR : Untranslated Region.
}

\section{Supplementary Information}

The online version contains supplementary material available at https://doi. org/10.1186/s13148-021-01171-w.

Additional file 1: Supplementary Table 1. Characteristics of metabolic disease-related SNPS.

Additional file 2: Supplementary Table 2. Significant DM-CpGs from analyses with and without adjusting for cell composition.

Additional file 3: Supplementary Table 3. Characteristics of the 174 DM-CpGs.

Additional file 4: Supplementary Table 4. Distribution of DM-CpGs across functional genomic regions.
Additional file 5: Supplementary Table 5. Estimated correlation between DM-CpGs and corresponding transcripts.

Additional file 6: Supplementary Table 6 DM-CpG genes associated with liver function and liver tumorigenesis as identified by IPA.

Additional file 7: Supplementary Table 7. DM-CpGs association with metabolic disease-related SNPS.

Additional file 8: Supplementary Table 8. Correlation between DMCpGs and blood biomarkers (FDR $<0.1)$.

Additional file 9: Supplementary Table 9. Characteristics of TCGA-LIHC samples (40 pairs of tumor and adjacent normal tissue).

Additional file 10: Supplementary Table 10. Among 174 DM-CpGs, differential methylation between Asian and EUA in normal tissue from TCGA-LIHC dataset (FDR < 0.1).

Additional file 11: Supplementary Table 11. Among 174 DM-CpGs, differential methylation between tumor $(\mathrm{T})$ and adjacent normal $(\mathrm{N})$ tissues from TCGA-LIHC dataset (FDR < 0.1).

\section{Acknowledgements}

We wish to thank the Genomics and Bioinformatics Shared Resource (GBSR) at the University of Hawaii Cancer Center for performing the genomic analyses, especially Ms. Annette Jones for running the Human Methylation450K assays. We also thank the study participants and the staff for recruiting.

\section{Authors' contributions}

MAS and AES contributed equally to this paper. MAS and AES wrote the first draft of the manuscript. MAS and UL made substantial contributions to the conception and design of the work. MAS, AES, and KJA interpreted data and MAS, AES, KJA, and UL were significant contributors in writing the manuscript. MAS and AES analyzed data, and KJA oversaw the statistical data analysis. UL, KMM, SYP, LC, TE, MT, KP, LRW, and LLM all has significant to contribution to the design and evaluation of the data. As the corresponding author, MAS had full access to all of the data in the study and had final responsibility for the decision to submit for publication. All authors read, revised, and approved the manuscript.

\section{Funding}

This work was supported by the U.S. National Cancer Institute (NCI) for the University of Hawaii Cancer Center (P30 CA071789; MT, LRW, LLM, and UL) and NCI Program Project Grant (P01 CA168530; LLM, UL, LRW, MT), a research startup fund (MAS) provided by OSU College of Public Health, a research start-up fund (KJA) from OSUCCC, and a U.S. National Institutes of Health (NIH) Grant (3U01CA164973-09S1; MAS, KJA, UL, LLM, LRW).

Availability of data and materials

The datasets generated during and/or analyzed during the current study are available from the corresponding author and the MEC Research Committee on individual requests.

\section{Declarations}

\section{Ethics approval and consent to participate}

The study was approved by the Institutional Review Boards of the University of Hawaii and the Queen's Medical Center, Honolulu, and all participants signed an informed consent.

\section{Consent for publication}

Not applicable.

\section{Competing interests}

The authors declare that they have no competing interests.

\section{Author details}

${ }^{1}$ Division of Environmental Health Science, College of Public Health, The Ohio State University, 404 Cunz Hall, 1841 Neil Ave., Columbus, OH 43210, USA.

${ }^{2}$ Division of Biostatistics, College of Public Health, The Ohio State University, Columbus, OH, USA. ${ }^{3}$ Population Sciences in the Pacific Program, University 
of Hawaii Cancer Center, University of Hawaii at Manoa, Honolulu, HI, USA. ${ }^{4}$ School of Medicine, University of Maryland, Baltimore, MD, USA. ${ }^{5}$ Genomics and Bioinformatics Shared Resources, University of Hawaii Cancer Center, University of Hawaii at Manoa, Honolulu, HI, USA.

Received: 25 May 2021 Accepted: 25 August 2021

Published online: 11 October 2021

\section{References}

1. Krishnadath IS, Toelsie JR, Hofman A, Jaddoe VW. Ethnic disparities in the prevalence of metabolic syndrome and its risk factors in the Suriname Health Study: a cross-sectional population study. BMJ Open. 2016;6(12):e013183.

2. Acciai F, Noah AJ, Firebaugh G. Pinpointing the sources of the Asian mortality advantage in the USA. J Epidemiol Community Health. 2015;69(10):1006-11.

3. Palaniappan LP, Wong EC, Shin JJ, Fortmann SP, Lauderdale DS. Asian Americans have greater prevalence of metabolic syndrome despite lower body mass index. Int J Obes (Lond). 2011;35(3):393-400.

4. Kolonel LN, Henderson BE, Hankin JH, Nomura AM, Wilkens LR, Pike MC, Stram DO, Monroe KR, Earle ME, Nagamine FS. A multiethnic cohort in Hawaii and Los Angeles: baseline characteristics. Am J Epidemiol. 2000;151(4):346-57.

5. Maskarinec G, Grandinetti A, Matsuura G, Sharma S, Mau M, Henderson $\mathrm{BE}$, Kolonel LN. Diabetes prevalence and body mass index differ by ethnicity: the Multiethnic Cohort. Ethn Dis. 2009;19(1):49-55.

6. Lim U, Monroe KR, Buchthal S, Fan B, Cheng I, Kristal BS, Lampe JW, Hullar MA, Franke AA, Stram DO et al. Propensity for intra-abdominal and hepatic adiposity varies among ethnic groups. Gastroenterology 2019;156(4):966-975.

7. Setiawan VW, Stram DO, Porcel J, Lu SC, Le Marchand L, Noureddin M. Prevalence of chronic liver disease and cirrhosis by underlying cause in understudied ethnic groups: the multiethnic cohort. Hepatology (Baltimore, MD). 2016;64(6):1969-77.

8. Setiawan VW, Lim U, Lipworth L, Lu SC, Shepherd J, Ernst T, Wilkens LR, Henderson BE, Le Marchand L. Sex and ethnic differences in the association of obesity with risk of hepatocellular carcinoma. Clin Gastroenterol Hepatol. 2016;14(2):309-16.

9. Pham C, Fong TL, Zhang J, Liu L. Striking racial/ethnic disparities in liver cancer incidence rates and temporal trends in California, 1988-2012. J Natl Cancer Inst. 2018;110(11):1259-69.

10. Xu R, Tao A, Zhang S, Deng Y, Chen G. Association between patatin-like phospholipase domain containing 3 gene (PNPLA3) polymorphisms and nonalcoholic fatty liver disease: a HuGE review and meta-analysis. Sci Rep. 2015;5:9284.

11. Park SL, Li Y, Sheng X, Hom V, Xia L, Zhao K, Pooler L, Setiawan VW, Lim U, Monroe KR, et al. Genome-wide association study of liver fat: the multiethnic cohort adiposity phenotype study. Hepatol Commun. 2020;4(8):1112-23.

12. Xia YY, Ding YB, Liu XQ, Chen XM, Cheng SQ, Li LB, Ma MF, He JL, Wang YX. Racial/ethnic disparities in human DNA methylation. Biochim Biophys Acta. 2014;1846(1):258-62.

13. Galanter JM, Gignoux CR, Oh SS, Torgerson D, Pino-Yanes M, Thakur N, Eng C, Hu D, Huntsman S, Farber HJ et al. Differential methylation between ethnic sub-groups reflects the effect of genetic ancestry and environmental exposures. Elife 2017, 6.

14. Jaenisch R, Bird A. Epigenetic regulation of gene expression: how the genome integrates intrinsic and environmental signals. Nat Genet. 2003;33(Suppl):245-54.

15. Gomez-Alonso MDC, Kretschmer A, Wilson R, Pfeiffer L, Karhunen V, Seppala I, Zhang W, Mittelstrass K, Wahl S, Matias-Garcia PR, et al. DNA methylation and lipid metabolism: an EWAS of 226 metabolic measures Clin Epigenet. 2021;13(1):7.

16. Terry MB, Ferris JS, Pilsner R, Flom JD, Tehranifar P, Santella RM, Gamble MV, Susser E. Genomic DNA methylation among women in a multiethnic New York City birth cohort. Cancer Epidemiol Biomark Prev. 2008;17(9):2306-10.

17. Zhang FF, Cardarelli R, Carroll J, Fulda KG, Kaur M, Gonzalez K, Vishwanatha JK, Santella RM, Morabia A. Significant differences in global genomic
DNA methylation by gender and race/ethnicity in peripheral blood. Epigenetics. 2011;6(5):623-9.

18. Fraser HB, Lam LL, Neumann SM, Kobor MS. Population-specificity of human DNA methylation. Genome Biol. 2012;13(2):R8.

19. Kim KC, Friso S, Choi SW. DNA methylation, an epigenetic mechanism connecting folate to healthy embryonic development and aging. J Nutr Biochem. 2009;20(12):917-26.

20. Lim U, Ernst T, Buchthal SD, Latch M, Albright CL, Wilkens LR, Kolonel LN, Murphy SP, Chang L, Novotny R, et al. Asian women have greater abdominal and visceral adiposity than Caucasian women with similar body mass index. Nutr Diabetes. 2011;1:e6.

21. Song MA, Ernst T, Tiirikainen M, Tost J, Wilkens LR, Chang L, Kolonel LN, Le Marchand L, Lim U. Methylation of imprinted IGF2 regions is associated with total, visceral, and hepatic adiposity in postmenopausal women. Epigenetics. 2018;13(8):858-65.

22. Team RC: R: A Language and Environment for Statistical Computing. In. Vienna, Austria: R Foundation for Statistical Computing; 2018.

23. Huber W, Carey VJ, Gentleman R, Anders S, Carlson M, Carvalho BS, Bravo HC, Davis S, Gatto L, Girke T, et al. Orchestrating high-throughput genomic analysis with Bioconductor. Nat Methods. 2015;12(2):115-21.

24. Aryee MJ, Jaffe AE, Corrada-Bravo H, Ladd-Acosta C, Feinberg AP, Hansen KD, Irizarry RA. Minfi: a flexible and comprehensive bioconductor package for the analysis of Infinium DNA methylation microarrays. Bioinformatics (Oxford, England). 2014;30(10):1363-9.

25. Du P, Zhang X, Huang CC, Jafari N, Kibbe WA, Hou L, Lin SM. Comparison of beta-value and $M$-value methods for quantifying methylation levels by microarray analysis. BMC Bioinf. 2010;11:587.

26. Maksimovic J, Gordon L, Oshlack A. SWAN: Subset-quantile within array normalization for illumina infinium HumanMethylation450 BeadChips. Genome Biol. 2012;13(6):R44.

27. Chen YA, Lemire M, Choufani S, Butcher DT, Grafodatskaya D, Zanke BW, Gallinger S, Hudson TJ, Weksberg R. Discovery of cross-reactive probes and polymorphic CpGs in the Illumina Infinium HumanMethylation450 microarray. Epigenetics. 2013;8(2):203-9.

28. Price ME, Cotton AM, Lam LL, Farre P, Emberly E, Brown CJ, Robinson WP, Kobor MS. Additional annotation enhances potential for biologically-relevant analysis of the Illumina Infinium HumanMethylation450 BeadChip array. Epigenet Chromatin. 2013;6(1):4.

29. Gentry AE, Jackson-Cook CK, Lyon DE, Archer KJ. Penalized ordinal regression methods for predicting stage of cancer in high-dimensional covariate spaces. Cancer Inform. 2015;14(Suppl 2):201-8.

30. Bozic T, Kuo CC, Hapala J, Franzen J, Eipel M, Platzbecker U, Kirschner $\mathrm{M}$, Beier $\mathrm{F}$, Jost $\mathrm{E}$, Thiede $\mathrm{C}$ et al. Investigation of measurable residual disease in acute myeloid leukemia by DNA methylation patterns. Leukemia 2021.

31. Johnson WE, Li C, Rabinovic A. Adjusting batch effects in microarray expression data using empirical Bayes methods. Biostatistics (Oxford, England). 2007:8(1):118-27.

32. Leek JT, Johnson WE, Parker HS, Jaffe AE, Storey JD. The sva package for removing batch effects and other unwanted variation in high-throughput experiments. Bioinformatics (Oxford, England). 2012;28(6):882-3.

33. Jaffe $A E$, Irizarry RA. Accounting for cellular heterogeneity is critical in epigenome-wide association studies. Genome Biol. 2014;15(2):R31.

34. Reinius LE, Acevedo N, Joerink M, Pershagen G, Dahlen SE, Greco D, Soderhall C, Scheynius A, Kere J. Differential DNA methylation in purified human blood cells: implications for cell lineage and studies on disease susceptibility. PLoS ONE. 2012;7(7):e41361.

35. Houseman EA, Accomando WP, Koestler DC, Christensen BC, Marsit CJ, Nelson HH, Wiencke JK, Kelsey KT. DNA methylation arrays as surrogate measures of cell mixture distribution. BMC Bioinf. 2012;13:86.

36. Van den Boogaart KG, Tolosana-Delgado R. Analyzing compositional data with R, vol. 122. Berlin: Springer; 2013.

37. Carvalho BS, Irizarry RA. A framework for oligonucleotide microarray preprocessing. Bioinformatics (Oxford, England). 2010;26(19):2363-7.

38. Irizarry RA, Bolstad BM, Collin F, Cope LM, Hobbs B, Speed TP. Summaries of affymetrix GeneChip probe level data. Nucleic Acids Res. 2003;31(4):e15

39. Bolstad BM, Irizarry RA, Astrand M, Speed TP. A comparison of normalization methods for high density oligonucleotide array data based on variance and bias. Bioinformatics (Oxford, England). 2003;19(2):185-93. 
40. Irizarry RA, Hobbs B, Collin F, Beazer-Barclay YD, Antonellis KJ, Scherf U, Speed TP. Exploration, normalization, and summaries of high density oligonucleotide array probe level data. Biostatistics (Oxford, England). 2003:4(2):249-64.

41. MacDonald JW: hta20transcriptcluster.db: Affymetrix hta20 annotation data (chip hta20transcriptcluster). In., vol. Version 8.7.0: R Package; 2017.

42. Lim U, Turner SD, Franke AA, Cooney RV, Wilkens LR, Ernst T, Albright CL, Novotny R, Chang L, Kolonel LN, et al. Predicting total, abdominal, visceral and hepatic adiposity with circulating biomarkers in Caucasian and Japanese American women. PLoS ONE. 2012;7(8):e43502.

43. Ritchie ME, Phipson B, Wu D, Hu Y, Law CW, Shi W, Smyth GK. limma powers differential expression analyses for RNA-sequencing and microarray studies. Nucleic Acids Res. 2015;43(7):e47.

44. Hannum G, Guinney J, Zhao L, Zhang L, Hughes G, Sadda S, Klotzle B, Bibikova M, Fan JB, Gao Y, et al. Genome-wide methylation profiles reveal quantitative views of human aging rates. Mol Cell. 2013;49(2):359-67.

45. Vick AD, Burris HH. Epigenetics and Health Disparities. Curr Epidemiol Rep. 2017:4(1):31-7.

46. Horvath S, Gurven M, Levine ME, Trumble BC, Kaplan H, Allayee H, Ritz BR, Chen B, Lu AT, Rickabaugh TM, et al. An epigenetic clock analysis of race/ ethnicity, sex, and coronary heart disease. Genome Biol. 2016;17(1):171.

47. He F, Berg A, Imamura Kawasawa Y, Bixler EO, Fernandez-Mendoza J, Whitsel EA, Liao D. Association between DNA methylation in obesityrelated genes and body mass index percentile in adolescents. Sci Rep. 2019;9(1):2079.

48. Richard MA, Huan T, Ligthart S, Gondalia R, Jhun MA, Brody JA, Irvin MR, Marioni R, Shen J, Tsai PC, et al. DNA methylation analysis identifies loci for blood pressure regulation. Am J Hum Genet. 2017;101 (6):888-902.

49. Zhang JY, Weng MZ, Song FB, Xu YG, Liu Q, Wu JY, Qin J, Jin T, Xu JM. Long noncoding RNA AFAP1-AS1 indicates a poor prognosis of hepatocellular carcinoma and promotes cell proliferation and invasion via upregulation of the RhoA/Rac2 signaling. Int J Oncol. 2016;48(4):1590-8.

50. Ikeda A, Shimizu T, Matsumoto Y, Fujii Y, Eso Y, Inuzuka T, Mizuguchi A, Shimizu K, Hatano E, Uemoto $S$, et al. Leptin receptor somatic mutations are frequent in HCV-infected cirrhotic liver and associated with hepatocellular carcinoma. Gastroenterology. 2014;146(1):222-32.

51. Leow SC, Poschmann J, Too PG, Yin J, Joseph R, McFarlane C, Dogra S, Shabbir A, Ingham PW, Prabhakar S, et al. The transcription factor SOX6 contributes to the developmental origins of obesity by promoting adipogenesis. Development. 2016;143(6):950-61.

52. Beckers S, Zegers D, Van Gaal LF, Van Hul W. Replication of the SH2B1 rs7498665 association with obesity in a Belgian study population. Obes Facts. 2011:4(6):473-7.

53. Sheng L, Liu Y, Jiang L, Chen Z, Zhou Y, Cho KW, Rui L. Hepatic SH2B1 and $\mathrm{SH} 2 \mathrm{~B} 2$ regulate liver lipid metabolism and VLDL secretion in mice. PLoS ONE. 2013;8(12):e83269.
54. Hotta K, Kitamoto T, Kitamoto A, Mizusawa S, Matsuo T, Nakata Y, Hyogo $\mathrm{H}$, Ochi H, Kamohara S, Miyatake N, et al. Computed tomography analysis of the association between the SH2B1 rs7498665 single-nucleotide polymorphism and visceral fat area. J Hum Genet. 2011;56(10):716-9.

55. BasuRay S, Wang Y, Smagris E, Cohen JC, Hobbs HH. Accumulation of PNPLA3 on lipid droplets is the basis of associated hepatic steatosis. Proc Natl Acad Sci USA. 2019;116(19):9521-6.

56. Trepo E, Romeo S, Zucman-Rossi J, Nahon P. PNPLA3 gene in liver diseases. J Hepatol. 2016;65(2):399-412.

57. Willer CJ, Speliotes EK, Loos RJ, Li S, Lindgren CM, Heid IM, Berndt SI, Elliott AL, Jackson AU, Lamina C, et al. Six new loci associated with body mass index highlight a neuronal influence on body weight regulation. Nat Genet. 2009:41(1):25-34.

58. Lamiquiz-Moneo I, Mateo-Gallego R, Bea AM, Dehesa-Garcia B, PerezCalahorra S, Marco-Benedi V, Baila-Rueda L, Laclaustra M, Civeira F, Cenarro A. Genetic predictors of weight loss in overweight and obese subjects. Sci Rep. 2019;9(1):10770.

59. Cheung CY, Tso AW, Cheung BM, Xu A, Ong KL, Fong CH, Wat NM, Janus $E D$, Sham PC, Lam KS. Obesity susceptibility genetic variants identified from recent genome-wide association studies: implications in a chinese population. J Clin Endocrinol Metab. 2010;95(3):1395-403.

60. Chaisaingmongkol J, Budhu A, Dang H, Rabibhadana S, Pupacdi B, Kwon SM, Forgues M, Pomyen Y, Bhudhisawasdi V, Lertprasertsuke N, et al. Common molecular subtypes among asian hepatocellular carcinoma and cholangiocarcinoma. Cancer Cell. 2017;32(1):57-70.

61. Lossie AC, Muir WM, Lo CL, Timm F, Liu Y, Gray W, Zhou FC. Implications of genomic signatures in the differential vulnerability to fetal alcohol exposure in C57BL/6 and DBA/2 mice. Front Genet. 2014;5:173.

62. Chiang KM, Chang $\mathrm{HC}$, Yang $\mathrm{HC}$, Chen $\mathrm{CH}$, Chen HH, Lee WJ, Pan WH. Genome-wide association study of morbid obesity in Han Chinese. BMC Genet. 2019:20(1):97.

63. Kim YM, Stone M, Hwang TH, Kim YG, Dunlevy JR, Griffin TJ, Kim DH. SH3BP4 is a negative regulator of amino acid-Rag GTPase-mTORC1 signaling. Mol Cell. 2012;46(6):833-46.

64. Sadeghi M, Lahdou I, Oweira H, Daniel V, Terness P, Schmidt J, Weiss KH, Longerich T, Schemmer P, Opelz G, et al. Serum levels of chemokines CCL4 and CCL5 in cirrhotic patients indicate the presence of hepatocellular carcinoma. Br J Cancer. 2015;113(5):756-62.

65. Seki E, De Minicis S, Gwak GY, Kluwe J, Inokuchi S, Bursill CA, Llovet JM, Brenner DA, Schwabe RF. CCR1 and CCR5 promote hepatic fibrosis in mice. J Clin Investig. 2009;119(7):1858-70.

\section{Publisher's Note}

Springer Nature remains neutral with regard to jurisdictional claims in published maps and institutional affiliations.
Ready to submit your research? Choose BMC and benefit from:

- fast, convenient online submission

- thorough peer review by experienced researchers in your field

- rapid publication on acceptance

- support for research data, including large and complex data types

- gold Open Access which fosters wider collaboration and increased citations

- maximum visibility for your research: over $100 \mathrm{M}$ website views per year

At BMC, research is always in progress.

Learn more biomedcentral.com/submissions 